\title{
MIRADA RETROSPECTIVA A LA INSEMINACIÓN ARTIFICIAL EN OVINOS
}

\section{A RETROSPECTIVE LOOK AT THE ARTIFICIAL INSEMINATION IN SHEEP}

\author{
(iD) René Antonio Hinojosa Benavides ${ }^{*}$, (D) Carmen Thalia Andia Salinas ${ }^{1}$, (D) Raúl Lunazco \\ Gamboa $^{1}$, Katy Núñez Quispe ${ }^{1}$, (D) Walter Miguel Ruiz Vilca ${ }^{1}$, (D) Rubria Farfán Mauri", \\ (iD) Jhon Vitor Valladolid ${ }^{1}$
}

rhinojosa@unah.edu.pe; thaliaandiasalinas100@gmail.com; raullunazco25@gmail.com; katyNQ2806@gmail.com; thaliaandiasalinas100@gmail.com; rubriafarfanmauri@gmail.com; Mkey40@hotmail.com ${ }^{1}$ Universidad Nacional Autónoma de Huanta, Ayacucho, Perú

*Correspondencia: René Antonio Hinojosa Benavides. Email: rhinojosa@unah.edu.pe

Recibido: 10.06.19 | Aprobado: 17.06.19

\section{RESUMEN}

El objetivo fue analizar el efecto de esta biotecnología reproductiva en la producción de ovinos, principalmente en aspectos fundamentales como: eficiencia reproductiva, mejoramiento genético, transferencia de tecnología al campo y capacitación del productor; todo finalmente reflejado en la productividad y la rentabilidad de la producción ovina, teniéndose en cuenta también todos los factores que afectan al correcto desarrollo de la inseminación artificial, evitando los momentos de más calor o frío ambiental y practicando el flushing si el estado general de las ovejas lo aconseja. Se concluyó que las diferencias entre los resultados obtenidos por las diferentes explotaciones son altamente significativas.

Palabras clave: Biotecnología, genes, mejoramiento genético.

\section{ABSTRACT}

The aim was to analyze the effect that this reproductive biotechnology on the production of sheep, mainly on fundamental aspects such as the reproductive efficiency, the genetic improvement, the technology transfer to the field and training of the producer, everything finally reflected in the productivity and the profitability of sheep production, taking into account also all the factors that affect the correct development of artificial insemination, avoiding the moments of more heat or environmental cold and practicing the flushing if the general state of the She advises it. It was concluded that the differences between the results obtained by the different farms are highly significant.

Keywords: Biotechnology. Genes. Genetic improvement. 


\section{INTRODUCCIÓN}

Desde la década de los '90 ha aumentado el interés en el uso de la Inseminación Artificial (IA) en los ovinos, con el fin de mejorar los sistemas de explotación, así como la calidad genética de la especie (Angulo et al. 1999). Ramírez et al. (2005) mencionaron que las IA se hacen sobre estros detectados; sin embargo, uno de los objetivos de la sincronización de celos es realizar inseminaciones a tiempo fijo para reducir el costo de mano de obra, el uso de receladores y de manejo de animales, sobre todo al inseminar rebaños numerosos. Para que una explotación ovina sea productiva se requiere que una oveja tenga al menos tres partos en dos años; para ello, el desarrollo de una gran variedad de protocolos de sincronización de estros a base de hormonas esteroidales y no esteroidales, diluyentes mejorados y de la inseminación laparoscópica con semen congelado, han permitido obtener porcentajes de concepción al primer servicio hasta del 78,57\% (Martínez et al., 2006).

La inseminación artificial (IA) en ovinos se inició a principios del siglo XX en Rusia con semen fresco el cual era depositado en el fondo de la vagina, obteniendo muy bajas tasas de fertilidad (5-30\%); al perfeccionarse la técnica surge la inseminación cervical o pericervical con semen fresco o refrigerado, la cual observa resultados que van del 10 al $60 \%$, debido a la diversidad morfológica en la anatomía del cérvix en esta especie. La ovinocultura requiere herramientas como la IA por laparoscopia, para poder establecer programas de mejoramiento genético eficientes. Tejedor et al. (2016) aseveraron que la comunicación entre técnicos y ganaderos es esencial puesto que todos los detalles del manejo relacionados con la IA (incluido el periodo anterior y posterior a la misma) deben optimizarse para garantizar el éxito de la IA.

La inseminación artificial es una técnica reproductiva para el progreso genético; sin embargo, la inseminación con semen congelado no es satisfactoria por la baja tasa de fertilidad, que puede ser prevenida con el uso de un dilutor adecuado (Ramos et al., 2017). Para dar solución a dicha situación se ha desarrollado la técnica de inseminación laparoscópica la cual permite un mejor acceso al útero de los ovinos, así como la valoración de la cavidad abdominal y por lo tanto la optimización del semen (Flores et al., 2017).

\section{Consideraciones Genéricas}

La especie ovina es poliéstrica estacional, lo que significa que su conducta reproductiva está ligada a las estaciones, específicamente al fotoperiodo, por lo tanto, se presenta un período de anestro durante una gran parte del año, afectando de esta manera la producción. Sin embargo, en Colombia como país tropical no se presenta este fenómeno tan marcado, aunque sí hay un pequeño descenso en las manifestaciones de estro durante los meses de marzo, abril y mayo (Porras et al., 2003).

La IA con semen congelado juega un rol muy importante en los programas de mejoramiento genético, no solo por acelerar el flujo de material genético superior hacia sectores de inferiores características productivas, sino por facilitar el transporte de semen, evitando el costoso traslado de los reproductores y disminuyendo los riesgos sanitarios. No obstante, para poder cumplir con el mejoramiento genético, es fundamental que la técnica reproductiva garantice adecuadas tasas de preñez (Mellisho et al., 2006). 
La reproducción en pequeños rumiantes puede ser controlada por diversos protocolos de sincronización. Algunos de estos métodos involucran la administración de hormonas que modifican la cadena de eventos durante el ciclo estral. En los protocolos de sincronización son usados la progesterona o sus análogos, entre los cuales los más usados son los progestágenos, los cuales imitan la función del cuerpo lúteo (CL). Por otra parte, el uso de las prostaglandinas es una alternativa para controlar la reproducción eliminando el CL e induciendo una subsecuente fase folicular acompañada de ovulación. Adicionalmente, la gonadotrofina coriónica equina (eCG), siendo una hormona análoga a la LH (hormona luteinizante), ayuda con la presentación de la ovulación.

Por su parte, la hormona liberadora de gonadotropina ( $\mathrm{GnRH}$ ) estimula la producción de las hormonas folículo estimulante (FSH) y de la LH. El uso de hormonas para inducir el estro ha permitido implementar la inseminación artificial (IA) en hembras ovinas y programas de ovulación múltiple y transferencia de embriones (LozanoGonzález et al., 2012).

Los ovinos al igual que otros animales domésticos, no son originarios del continente americano, sin embargo, presentan una amplia distribución geográfica, debido a su gran capacidad de adaptación a las diferentes condiciones de clima, vegetación y manejo (Ocampo, 2014). La IA es una alternativa importante para el mejoramiento genético y productivo del hato (Aké-López et al., 2014b). La progesterona (P4) tiene funciones muy importantes, controlando la secreción maternal de nutrientes, además de la regulación de factores de crecimiento y agentes inmunosupresivos que son necesarios para el desarrollo del embrión y el reconocimiento materno de la preñez, de este modo, se ha establecido que la presencia de la $\mathrm{P} 4$ es esencial para las ovejas principalmente durante las primeras semanas de gestación (Torres-Lemus et al., 2018). La inseminación artificial en el ovino ha despertado el interés de los ganaderos, con el fin de mejorar los sistemas de explotación y la calidad genética del rebaño (Cabrera et al., 2011).

\section{Importancia de la Inseminación Artificial}

La importancia de la inseminación artificial al inicio de un programa de mejoramiento, radica en que permite la planificación de las estrategias de conexión genética de los rebaños utilizando sementales de referencia. Posteriormente, su importancia es mayor en la difusión del mejoramiento en los rebaños de estratos más bajos mediante la utilización de sementales probados. En un diagnóstico sobre la ovinocultura se detectó que dentro de las líneas estratégicas de investigación que se deben abordar, se encuentra la baja disponibilidad de animales mejoradores, por lo que es fundamental el esfuerzo de los criadores para evaluar e identificar animales que sean mejoradores de las características productivas con importancia económica (Quiroz, 2012). El éxito de la IA en ganado ovino se basa en los valores obtenidos de fecundidad, fertilidad, y prolificidad (Tejedor et al., 2016).

Las ventajas de la IA se incrementan con la disponibilidad de carneros de alto valor genético y con la posibilidad de congelar el semen para permitir un uso más racional. Esto último permite magnificar el uso de los sementales, disminuir los costos de producción y poder adquirir machos de calidad genética comprobada (Cabrera et al., 2011). 


\section{Influencia de Factores ambientales}

Los factores meteorológicos afectan a la fertilidad tras la IA en ovinos, de forma que las temperaturas no extremas la favorecían. En otras especies de rumiantes se han obtenido resultados similares. Se han detectado numerosos factores con efectos significativos sobre el éxito de la inseminación artificial: identidad del macho, ganadería, mes de la inseminación, temperatura ambiental, circunstancias ambientales, realización o no de flushing, manejo durante la inseminación, desarrollo de la inseminación, técnico inseminador, centro de recogida de semen, año, día tras el parto; en su mayoría se trata de efectos de pequeña magnitud, pero cada uno de ellos explica por separado una pequeña parte de la variación en el éxito de la IA. El balance coste/beneficio de realizar cambios sólo sería cuantificable en el caso del flushing (Tejedor et al., 2016). Diferentes estudios han identificado varios factores que reducen la eficiencia reproductiva de los ovinos: Raza, nutrición, edad, estación del año, manejo, lactación y las enfermedades parecen ser los más importantes (Martínez et al., 2006).

\section{Sincronización del Estro}

La sincronización del estro (SE) es una estrategia de manejo reproductivo que permite agrupar la presentación de estros, cubriciones, programar partos en una época prevista de antemano, también para programar destetes (Aké-López et al., 2014b). En los ovinos puede ser una herramienta de gran utilidad para proyectar la producción del rebaño, programando temporadas de empadres y de partos en forma estratégica, inducir la actividad ovárica en ovejas en anestro, optimizar la mano de obra entre otras cosas, así como también puede ser utilizada para usar más eficientemente biotecnologías como la inseminación artificial o la transferencia de embriones. Para sincronizar el estro se usan diferentes hormonas o combinaciones de ellas, entre éstas se encuentran la prostaglandina F2 $\alpha$ y los progestágenos (Aké-López et al., 2014a)

Así mismo, Aké-López et al., (2014a) indicaron también que a pesar de las diferentes ventajas que ofrece la sincronización del estro, su utilización en el ga-nado ovino no es muy difundida, entre las razones que se mencionan están: no todos los animales que responden al tratamiento presentan conducta estral, y de los que presentan estro no todos conciben al recibir monta o al ser inseminados, esto posiblemente debido a la falta de ovulación, o el desarrollo de un cuerpo lúteo de vida media corta. Tal vez, la razón más importante es el aspecto económico, ya que los costos para la sincronización del estro son relativamente altos y más aún cuando los resultados no son satisfactorios. Una opción que puede ser utilizada para disminuir los costos de los tratamientos basados en progestágenos, es la utilización de implantes reciclados (previamente utilizados en vacas), o bien usar la mitad de un implante nuevo por cada animal, lo cual redundaría en menor costo por tratamiento, sin embargo, no se tiene mucha información al respecto y la existente es muy antigua.

Una producción eficiente de carne, leche, lana y pie de cría depende de la eficiencia reproductiva de las hembras, es por ello que la investigación se enfoca a mejorarla con programas de sincronización de estros con progestágenos, metodología que brinda la posibilidad de aplicar la inseminación artificial. Para realizar la sincronización se han utilizado los progestágenos, se han efectuado diferentes protocolos modificando dosis, tiempos y vías de administración, sin embargo, aún no se ha determinado cual presenta 
mayor eficiencia con relación a los índices de concepción y preñez, manifestación de celos, así como la presentación de reacciones adversas (Urete y Porras, 2013).

\section{Momento y Eficiencia de la Inseminación Artificial}

Una opción es realizar la IA a tiempo fijo o sistemática (sin detección de estros) en el total de las ovejas. Se efectúa entre las 58 a 62 horas de finalizado el tratamiento de sincronización de estros (esponjas con progestágenos durante 14 días + 200 UI eCG al retirar las esponjas). Una segunda opción es realizar la IA en las ovejas detectadas en estro, entre las 36-72 horas de finalizado el tratamiento de sincronización (esponjas + eCG). Las ovejas detectadas en estro post retiro de las esponjas a las 36, 48 y 60 horas, se inseminan 12 horas post detección de estro y las que presentan comportamiento estral a las 72 horas se inseminan ni bien son detectadas en celo. Otra alternativa es "dejar pasar" los celos post tratamiento de sincronización de estros e inseminar sobre el celo retorno, que se presenta entre los 18 a 21 días de finalizado el tratamiento. Se realizan dos detecciones diarias de estros y se insemina a las 12 horas post detección de celos (Gibbons y Cueto, 2009).

\section{Calidad seminal y métodos de colecta}

Los espermatozoides de los mamíferos sufren algunos cambios estructurales y bioquímicos al recorrer el tubo genital de la hembra, que los habilitan para fertilizar al óvulo. Este proceso es la capacitación espermática (CE) y es indispensable para que ocurra la fertilización. La refrigeración y criopreservación de los espermatozoides reducen o interrumpen su actividad metabólica y alargan su viabilidad, pero la capacitación espermática (CE) inducida en el semen de carnero puede reducir la fertilidad (Rodríguez et al., 2008). La congelación del esperma del macho ovino plantea el uso de semen de alta calidad durante todo el proceso a fin de alcanzar mejores resultados, es decir, el mayor número posible de espermatozoides móviles y fecundantes después de la descongelación. Los diluyentes de semen deben contener agentes protectores para las membranas celulares durante el enfriamiento a $5{ }^{\circ} \mathrm{C}$ (generalmente yema de huevo) La adición de yema de huevo al dilutor tiene un efecto benéfico sobre el porcentaje de motilidad, particularmente después de un rápido enfriamiento del semen (Cabrera et al., 2011).

La técnica de colección del semen es muy importante, puesto que determina, en gran parte, la cantidad y calidad del semen colectado. Los carneros se pueden colectar con electroeyaculador y vagina artificial, más en la práctica, se utiliza esta última, ya que permite obtener eyaculados con buena concentración espermática Para colectar semen mediante vagina artificial los carneros necesitan de entrenamiento, y la manera más práctica es utilizar como estímulo hembras en estro y acostumbrar a los machos a la presencia y manipulación por parte del humano. Los machos también pueden aprender a montar hembras que no se encuentren en celo, lo que facilita su manejo y evita la necesidad de contar con hembras que se manipulen hormonalmente; sin embargo, para lograrlo, se necesita de un proceso mucho más largo y de la utilización de algunas técnicas de habituación (Canizales y Rodríguez, 2012).

La evaluación seminal estándar, incluye varios parámetros que se han agrupado en características macroscópicas y microscópicas. En las primeras se cuentan el aspecto, el color, el pH y el volumen; en la segunda la concentración, la movilidad, la vitalidad, la 
morfología, pruebas de integridad de las membranas plasmática y acrosomal, y las determinaciones de trayectorias y velocidades de movimiento (Ebel, 2012). La calidad seminal del eyaculado varía entre individuos, entre eyaculados del mismo individuo, con la estación del año y con el método de colecta de semen utilizado. En la especie ovina las técnicas de colecta seminal de uso más difundido son la vagina artificial (VA) y la electroeyaculación (EE). La VA es un método que se asemeja al servicio natural y de fácil aplicación, pero que requiere de un periodo previo de entrenamiento de los animales (Ledesma et al., 2013).

Diversas metodologías han sido propuestas para la evaluación de la capacidad reproductiva de los machos ovinos (carneros) las cuales incluyen la inspección físicosanitaria del animal y la evaluación seminal. Esta última, es una herramienta utilizada principalmente para determinar la capacidad de fertilización del espermatozoide, y por ende predecir el potencial fértil de un reproductor. Además, permite la selección de eyaculados para procesos de refrigeración o de congelación y la clasificación de los machos para la utilización en programas de monta directa o para la inseminación artificial. Sin embargo, esta evaluación no se emplea de manera cotidiana en el proceso de selección de machos reproductores en la producción ovina (Carrillo y Hernández, 2016).

\section{CONCLUSIONES}

El conocimiento de la fisiología reproductiva de las hembras ovinas, las hormonas de interés reproductivo, los dispositivos y protocolos utilizados para la sincronización del estro y de la ovulación, son de gran importancia para el mejoramiento genético de la ovinocultura. Es necesario que, a partir del conocimiento teórico, se inicien investigaciones que determinen cuál o cuáles son los protocolos más efectivos en los sistemas productivos, condiciones medioambientales y sanitarias, teniendo en cuenta la importancia de los diversos factores ambientales sobre la inseminación artificial en ovinos.

La proporción de ovejas que ovulan es mayor cuando las ovejas son sincronizadas con esponjas intravaginales o con implantes reciclados. La aplicación de protocolos de evaluación seminal, como parámetro para la selección de machos reproductores, permite ser más estricto en cuanto a la selección y/o descarte de animales posiblemente mejoradores. El conocimiento de la fisiología reproductiva de las hembras ovinas, las hormonas de interés reproductivo, los dispositivos y protocolos utilizados para la sincronización del estro y de la ovulación, son de gran importancia para el mejoramiento genético de la ovinocultura.

\section{REFERENCIAS BIBLIOGRÁFICAS}

Angulo R. B., Ortiz A., Berruecos J. M., Feldman D. Valencia J. (1999). Motilidad y fertilidad del semen de carnero descongelado a dos diferentes ritmos de temperatura. Rev. Veterinaria México, vol. 30, núm. 3, julio-septiembre, 1999, pp. 265-268.

Aké-López J.R., Aké-Villanueva J.R., Centurión-Castro F.G. y Aké-Villanueva N.Y. (2014a). Sincronización del estro y tasa de ovulación de ovejas Pelibuey tratadas con esponjas intravaginales e implantes subcutáneos nuevos y reciclados. Rev. Bioagrociencias Vol. 7 N 1, enero-junio 2014. 
Aké-López J.R., Centurión-Castro F.G., Magaña-Monforte J.G., Aké-Villanueva J.R. (2014b). Efecto del progestágeno y de la dosis de gonadotropina corionica equina en la sincronización del estro y tasa de gestación en ovejas pelibuey inseminadas por laparoscopia. Rev. Ecosistemas y Recursos Agropecuarios 1(3):261-268,2014.

Cabrera P., Ayulo A. y Pantoja C. (2011). Efecto del dilutor TRIS y citrato con yema de huevo de cordorniz sobre la viabilidad espermática en semen ovino congelado en pajillas. Rev Inv Vet Perú 2011; 22 (2): 105-113.

Canizales S. y Rodríguez J. (2012). Adiestramiento de carneros para la colección de semen con vagina artificial. Revista Colombiana de Ciencia Animal, Vol. 5, No. 1, 2012.

Carrillo-González D. y Hernández D. (2016). Caracterización seminal de individuos ovinos criollos colombianos de pelo en el departamento de Sucre. Rev. Colombiana Cienc Anim 2016; 8(2):197-203.

Ebel F. (2012). Caracterización y pruebas de congelabilidad seminal en asnos Baudet du Poitou. Tesis de grado. Universidad Austral de Chile.

Gibbons A. y Cueto M. (2009). Inseminación Artificial con Semen Congelado en Ovinos. Revista Presencia No53-2009.

Ledesma A., Manes J., Alberio R. y Osbor F. (2013). ¿Es posible mejorar la fertilidad del semen ovino criopreservado mediante la adición de plasma seminal? Taurus, 59, 1621.

Lozano-González J.F., Uribe-Velásquez L.F. y Osorio J.H. (2012). Control hormonal de la reproducción en hembras ovinas (Ovis aries). Revista Veterinaria y Zootecnía. 2012; 6(2): 134-147.

Martínez J. J., Sánchez M. T., Bucio L., Rojo R., Mendoza G. D., Cordero J. L., Mejía O. (2006). Efecto de ECG e inseminación laparoscópica sobre el comportamiento reproductivo en ovejas F1 (damara $\times$ merino). Revista Científica, vol. XVI, núm. 1, enero, 2006, pp. 72-77.

Mellisho E., Pinazo R., Chauca L., Cabrera P. y Rivas V. (2006). Inseminación intrauterina Vía Laparoscópica de Ovejas Black Belly con Semen Congelado. Rev Inv Vet Perú 2006; 17 (2): 131-136.

Ocampo J.R. (2014). Caracterización genética de ovinos en Colombia por medio de marcadores microsatélites. Tesis de Maestría. Universidad de Antioquia. Colombia.

Porras A., Zarco L.A. y Valencia J. (2003). Estacionalidad reproductiva en ovejas. Ciencia Veterinaria, v.9, p.2-14, 2003.

Quiroz J., Guerrero G., Oliva J., Granados L., Barrón M. (2012). Evaluación Genética de Características de Crecimiento del Ovino Pelibuey en Tabasco, México. AICA 2 (2012) 355-360

Ramírez A. J., Martínez R. D., Mejía O., Soto R. (2005). Modificación de la técnica de inseminación artificial intrauterina mediante laparoscopía en ovejas Pelibuey. Rev. Agrociencia, vol. 39, núm. 6, noviembre-diciembre, 2005, pp. 589-593. 
Ramos L., Rojas A., Martínez Z. (2017). Efecto de dilutores y tiempos de equilibrio en la criopreservación de semen ovino (Ovis aries). Revista de Investigación e Innovación Agropecuaria y de Recursos Naturales, La Paz, vol.4, n², pág. 6371, Diciembre 2017. ISSN: 2518-6868.

Rodríguez-Almeida F.A., Ávila Cota C.O., Anchondo A., Sánchez-Ramírez B. y Jiménez J.A. (2008). Sperm capacitation induced by conservation of diluted, refrigerated, or frozen ram semen. Rev. Agrociencia 42: 399-406. 2008.

Tejedor M.T., Monteagudo L.V., Laviña A. y Macías A. (2016). Factores ambientales que influyen en el éxito de la inseminación artificial en la raza ovina Rasa Aragonesa. Rev. Arch. Zootec. 65 (251): 321-325.2016.

Torres-Lemus F., Sánchez-Torres M.T., Nieto-Aquino, R., Salinas-Ríos T., Figueroa Velasco J.L., Martínez-Aispuro J.A., Cordero-Mora J., Rodríguez-Ortega L.T., Vargas-Monter J., Campero-Cruz A., Noriega-Trinidad V. (2018). Influencia del aporte exógeno de progesterona (CIDR) post-inseminación artificial en ovejas primalas. Rev. Agroproductividad: Vol. 11, Núm. 6, junio. 2018. pp: 114119.

Urete O., Porras J.L. (2013). Comparación de dos tratamientos a base de progestágenos para la sincronización de celos ovinos. Rev. Ciencia y Agricultura Vol. 10 - N. 22 Julio - Diciembre 2013, p.9-16 ISSN 0122-8420.

\section{CITAR COMO:}

Hinojosa Benavides, R. A., Andia Salinas, C. T., Lunazco Gamboa, R., Núñez Quispe, K., Ruíz Vilca, W. M., Farfán Mauri, R., \& Vitor Valladolid, J. (2019). Mirada retrospectiva a la inseminación artificial en ovinos. Puriq, 1(01), 83-90. https://doi.org/10.37073/puriq.1.01.14 